\title{
Two-colour flow-cytometric analysis of pulmonary alveolar macrophages from smokers
}

\author{
T. Umino*, C.M. Sköld', S.J. Pirruccello**, J.R. Spurzem*, S.I. Rennard*
}

\begin{abstract}
Two-colour flow-cytometric analysis of pulmonary alveolar macrophages from smokers. $T$. Umino, C.M. Sköld, S.J. Pirruccello, J.R. Spurzem, S.I. Rennard. (C)ERS Journals Ltd 1999.

ABSTRACT: The study of alveolar macrophages (AM) from smokers by flow cytometry (FCM) has been limited by strong autofluorescence and the lack of reliable markers to identify macrophages. Crystal violet quenching was reported to be effective in reducing autofluorescence of AM. CD68 is a marker for macrophages in immunohistochemistry, but has been less useful in FCM because of poor surface expression. This study evaluated the effectiveness of a method for two-colour FCM analysis of AM combined with membrane permeabilization and crystal violet quenching.
\end{abstract}

Bronchoalveolar lavage cells, fixed in $4 \%$ paraformaldehyde and permeabilized using $0.5 \%$ Triton X100, were incubated with fluorescent-labelled antibodies for 30 min and quenched with a saturated crystal violet solution. Two-colour FCM was then performed using forward/side scatter gating to select $\mathrm{AM}$.

Autofluorescence at $525 \mathrm{~nm}$ (fluorescein isothiocyanate) and $575 \mathrm{~nm}$ (phycoerythrin) markedly decreased after quenching. After permeabilization, $97.1 \pm 2.8 \%$ of the gated cells were $\mathrm{CD68}+$, while $53.9 \pm 18.6 \%$ of the $\mathrm{AM}$ were positive without permeabilization. CD68+ cells were sorted and proved to be AM morphologically. Analysis of CD71 (transferrin receptor) expression by FCM correlated with immunocytochemistry $(\mathrm{r}=0.77, \mathrm{p}<0.05)$.

The permeabilization/quenching technique, therefore, represents a satisfactory means to evaluate alveolar macrophages by flow cytometry. Eur Respir J 1999; 13: 894-899.

Inflammatory cells present in the pulmonary alveolar space can be readily sampled by bronchoalveolar lavage (BAL). The majority of these cells are macrophages, with smaller numbers of lymphocytes, neutrophils, eosinophils and basophils. Flow cytometry (FCM) has proven very useful for the study of the surface markers of lymphocytes in BAL fluid [1]. In contrast, FCM has been less useful for the analysis of macrophages, owing to the strong autofluorescence induced by phagocytosed dust particles, especially in smokers. This causes a high background fluorescence which masks immunofluorescent staining of the cells $[2,3]$. Analysis of BAL cells is further complicated by difficulty in separating cell types. With regard to size, large lymphocyte and epithelial cell populations can partially overlap. Two-colour analysis using a reliable marker to identify the cell type could be advantageous. CD68, for example, has been used as a marker to identify macrophages using immunohistochemical techniques. However, it has not been feasible to use CD68 in FCM analysis, because most of the CD68 antigen molecules are located intracellularly and few are expressed on the cell surface [4]. Thus, most of the studies on phenotypical markers of alveolar macrophages (AM) were based on immunocytochemical techniques [5].

\begin{abstract}
*Pulmonary and Critical Care Section, Dept of Internal Medicine, and **Dept of Pathology and Microbiology, University of Nebraska Medical Center, Omaha, NE, USA. ${ }^{+}$Division of Respiratory Medicine, Dept of Medicine, Karolinska Hospital, Stockholm, Sweden.
\end{abstract}

Correspondence: S.I. Rennard, Pulmonary and Critical Care Section, Dept of Internal Medicine, University of Nebraska Medical Center, 600 South 42nd Street, Omaha, NE 68198-5300, USA, Fax: 14025594878

Keywords: Bronchoalveolar lavage CD68

flow cytometry

macrophages

smoking

Received: April 161998

Accepted after revision October 201998

Funded by R.J. Reynolds. C.M. Sköld was funded, in part, by grants from the Swedish Council for Work Life Research, The Swedish Heart-Lung Foundation (King Oscar II Jubileumsfond) and the Swedish Society of Medicine.
Crystal violet quenching has been reported to effectively decrease the autofluorescence of AM, permitting singlecolour FCM analysis of the cells obtained from BAL $[6,7]$. Recent reports suggest that FCM for CD68 can be performed following appropriate membrane permeabilization $[8,9]$. Taking advantage of both these developments, this study sought to design a new method for a two-colour FCM analysis of AM. Permeabilization and intracellular crystal violet quenching were combined to investigate the feasibility of quantitative observation of surface and intracellular phenotypic antigens of AM, including those from smokers, using FCM. Such a method should greatly facilitate studies of the inflammatory cell populations of the alveolar space.

\section{Materials and methods}

\section{Bronchoalveolar lavage}

BAL cells were obtained from individuals participating in a research study approved by the University of Nebraska Institutional Review board. BAL was performed as described previously [10]. In brief, the bronchoscope was gently wedged into a segmental bronchus. Five aliquots of 
$20 \mathrm{~mL}$ normal saline were sequentially infused and immediately aspirated at each of the two sites. In general, two or three segments were lavaged. The return from the first aliquot at each site, representing largely bronchial material, was processed separately from the subsequent four aliquots from each site, which largely represented alveolar material. The "alveolar" samples from various sites were combined for further processing. The total number of cells recovered was determined using a haemocytometer. An aliquot was used to make a cytocentrifuge preparation, which was then

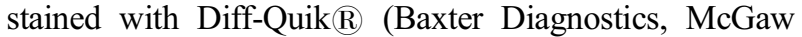
Park, IL, USA) in order to estimate the cell differential. If necessary, the sample was filtered through $250-\mu \mathrm{m}$ nylon mesh in order to remove mucus. The cells were then centrifuged $(300 \times g, 5 \mathrm{~min})$, after which they were suspended in phosphate-buffered saline (PBS) and used for FCM analysis.

\section{Immunofluorescent staining following permeabilization and quenching}

Approximately 500,000 BAL cells were placed in a 4$\mathrm{mL}$ tube. Cells were washed with $2 \mathrm{~mL}$ of PBS, centrifuged $(300 \times g, 5 \mathrm{~min})$ and fixed after resuspension in $0.5 \mathrm{~mL}$ of PBS containing $4 \%$ paraformaldehyde for $5 \mathrm{~min}$ at room temperature (RT). They were then washed with 2 $\mathrm{mL}$ of PBS, centrifuged $(300 \times g, 5 \mathrm{~min})$ and suspended in $0.2 \mathrm{~mL}$ of PBS containing $0.5 \%$ Triton X100 (Sigma, St Louis, MO, USA) for permeabilization. Twenty microlitres of human immunoglobulin $\left(10 \mathrm{mg} \cdot \mathrm{mL}^{-1}\right)$ was added to avoid subsequent nonspecific binding of monoclonal antibodies. After $10 \mathrm{~min}$ of incubation to permit permeabilization, fluorescent-labelled antibodies were added to the cell suspension and incubated for $30 \mathrm{~min}$ at RT. Fluorescein isothiocyanate (FITC)-labelled anti-CD68 antibody (clone KP-1) was purchased from DAKO USA (Carpinteria, CA, USA). As an example of a surface marker which reflects the functional status of AM, CD71 (transferrin receptor) was used. Phycoerythrin (PE)-labelled anti-CD71 antibody (clone YDJ1.2.2) and PE-labelled anti-human leukocyte antigen (HLA)-DR antibody (clone I3) were obtained from Beckman Coulter (Fullerton, CA, USA). The cells were washed with $2 \mathrm{~mL}$ of PBS, centrifuged $(300 \times g, 5 \mathrm{~min})$, suspended in $0.2 \mathrm{~mL}$ of saturated crystal violet solution (Certistain $\AA$; Merck, Darmstadt, Germany) and incubated for $5 \mathrm{~min}$ at RT to quench intracellular nonspecific fluorescence. The cells were then washed with $2 \mathrm{~mL}$ of PBS, centrifuged $(400 \times g, 8 \mathrm{~min})$ and resuspended in $0.5 \mathrm{~mL}$ of PBS. Control samples prepared without the permeabilization or the quenching procedure were prepared in parallel.

\section{Fluorescent microscopy}

Cytocentrifuge slides were prepared of permeabilized unstained cells with or without quenching, following the above procedure. These slides were observed under a fluorescent microscope with a 490-nm band-pass excitation filter and a 515-nm long-pass barrier filter.

\section{Flow-cytometric analysis}

The samples were evaluated with an Epics Elite $(\mathbb{R}$ flow cytometer (Beckman Coulter) equipped with an argon ion laser operating at $488 \mathrm{~nm}$ for excitation. A two-colour or three-colour analysis was performed, with a $525-\mathrm{nm}$ bandpass filter for FL-1 (FITC/green), 575-nm band-pass filter for FL-2 (PE/yellow) and 675-nm band-pass filter for FL-3 (Cy5/red). AM were gated by forward and side light scatter.

To observe the cytological features of selected cells, the cells were collected by fluorescence activated cell sorting with a FACStar plus $\AA$ flow cytometer (Becton \& Dickinson, San Jose, CA, USA) with a 485-nm argon laser for excitation. FITC was detected at $530 \mathrm{~nm}$ and PE was detected at $575 \mathrm{~nm}$. Sorted cells were prepared by cytocentrifuge and observed following Diff-Quik staining.

\section{Immunocytochemistry}

To compare the results obtained by FCM with an established method, CD71 staining was evaluated by both FCM analysis and immunocytochemistry. Cells from eight subjects over a range of CD71 expression as determined by FCM were selected for immunohistochemistry. An avidinbiotin complex (ABC)-alkaline phosphatase (AP) method was performed on the cytocentrifuge specimens. AntiCD71 (transferrin receptor) monoclonal antibody (clone DF1513; Sigma) was used as the primary antibody. Biotinylated horse antimouse immunoglobulin antibody (Vectastain $\mathbb{R}$; Vector Laboratories, Burlingame, CA, USA) was used as the secondary antibody. Streptavidin-alkaline phosphatase (Gibco BRL, Gaithersburg, MD, USA) was used to form ABC labelled by AP. A fast red/naphthol substrate kit (Sigma) was used for visualization. The percentage of macrophages that were CD71+ was determined on the cytocentrifuge specimens by counting 500 cells.

\section{Results}

\section{Effect of quenching to decrease autofluorescence}

When observed by fluorescence microscopy, AM from smokers had strong yellow autofluorescence in the absence of any fluorescent staining (fig. 1a). This changed to weak red fluorescence after the crystal violet quenching procedure (fig. 1b). Positive staining for CD68 could be readily observed in permeabilized quenched cells (fig. 1c). Corresponding results were obtained by FCM analysis (fig. 2). Autofluorescence intensity at $525 \mathrm{~nm}$ (FITC/green) and $575 \mathrm{~nm}$ (PE/yellow) was higher in AM from normal smokers (fig. $2 b$ and c) than in nonsmoker (fig. $2 \mathrm{f}$ and $\mathrm{g}$ ). The quenching procedure reduced the autofluorescence in AM from smokers so that it resembled that of the normal nonsmokers (fig. $2 \mathrm{~b}$ and c). These results were consistent for cells obtained from five smokers and five nonsmokers (table 1). In contrast, the fluorescence intensity at $675 \mathrm{~nm}$ (Cy5/red) increased after quenching (fig. $2 \mathrm{~d}$ and $\mathrm{h}$ ). 

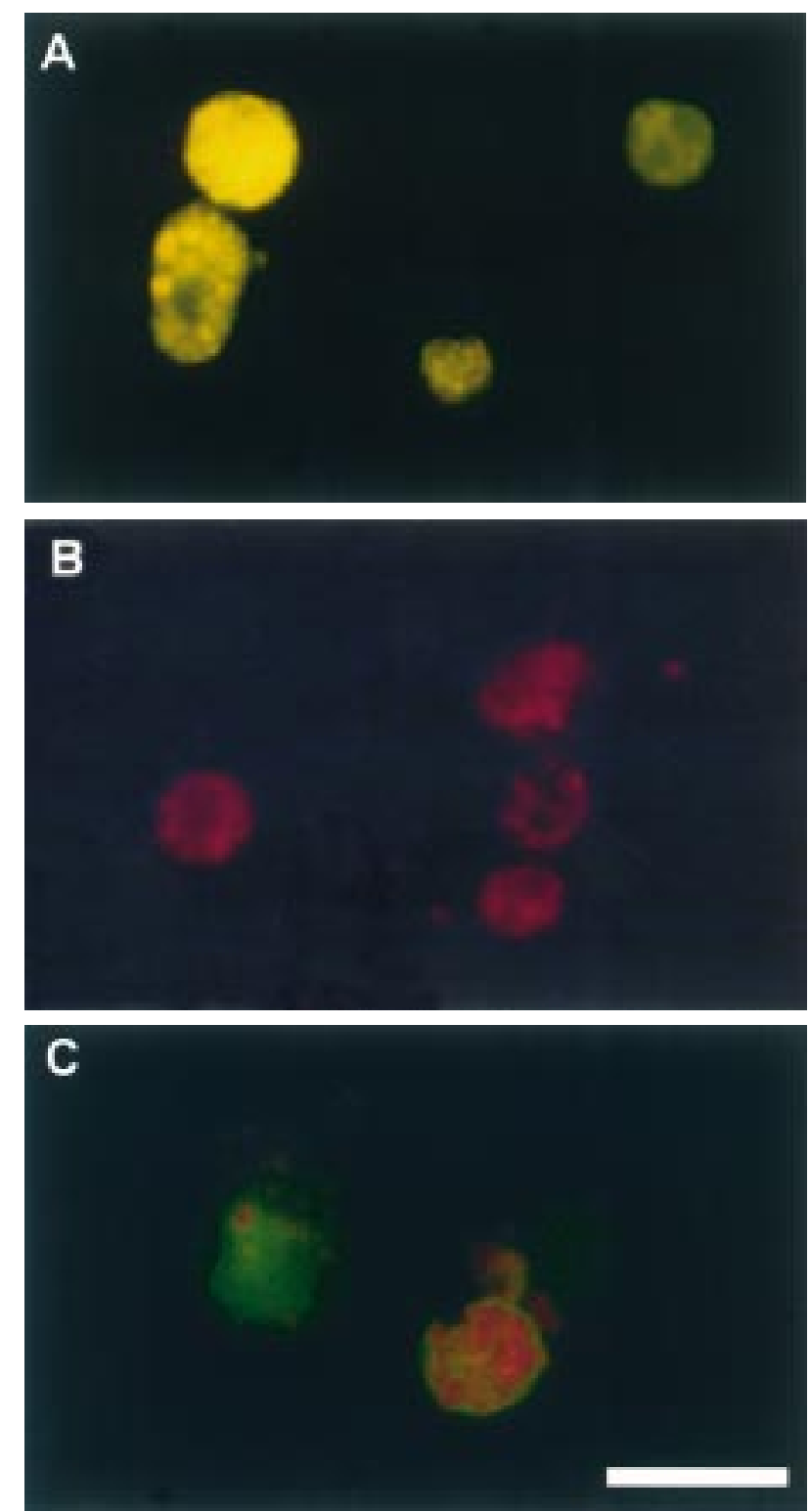

Fig. 1. - Effect of crystal violet quenching. Bronchoalveolar lavage cells were obtained from a 41-yr-old normal current smoker with a 30pack-yr smoking history. A) alveolar macrophages (AM) from the normal smoker had strong yellow autofluorescence (fixed and permeabilized cell without quenching). B) crystal violet quenching changed the autofluorescence to weak red (fixed, permeabilized and quenched cells). C) positive fluorescent stain by fluorescein isothiocyanate (FITC) (green) could be observed with quenched cells (permeabilized and quenched cells stained by FITC-labelled anti-CD68 antibody). (Internal scale bar $=50 \mu \mathrm{m}$.)

\section{Effect of permeabilization on CD68 staining}

Only a small percentage of AM were positive for CD68 in the absence of the permeabilization procedure (fig. 3c). However, most AM were positive for CD68 following the permeabilization procedure (fig. 3f). This result was consistently observed over four lavages analysed separately. As a group, $53.9 \pm 18.6 \%$ of AM were positive for CD68 without permeabilization, while $97.1 \pm 2.8 \%$ of AM were positive for CD68 following the permeabilization procedure. This was not due to nonspecific staining, as control immunoglobulin did not result in staining following the permeabilization procedure (fig. $3 \mathrm{~b}$ and e). Finally, the permeabilization procedure did not significantly affect the staining by antibodies directed against a surface antigen, HLA-DR (fig. $3 \mathrm{c}$ and $\mathrm{f}$ ). In the evaluation of the four cases, HLA-DR+ staining was $86.7 \pm 8.3 \%$ before and $83.9 \pm 8.8 \%$ after the permeabilization procedure $(\mathrm{p}=0.72)$.

To evaluate the reliability of macrophage selection by CD68 staining following permeabilization, FCM sorting was performed. The group of cells that was HLADR-positive and CD68-negative without permeabilization (quadrant (Q) 1 in fig. 3c) was morphologically identified as AM $(99.6 \%$ purity by 500 cell count) as well as the group that was HLA-DR-positive and CD68-positive (Q2 in fig. 3c) (100\% purity by 500 cell count). The group that was HLA-DR-positive and CD68-positive following permeabilization (Q6 in fig. 3f) was identified as AM with $100 \%$ purity in a 500 cell count.

Comparison between flow-cytometric analysis and immunocytochemistry

The percentage of macrophages which were CD71positive by FCM was compared with that by immunostaining of cytocentrifuge specimens of macrophages obtained from eight separate individuals. A statistically significant correlation was observed between the data obtained by the two methods using Pearson's correlation coefficient $(\mathrm{r}=0.77, \mathrm{p}<0.05)$ (fig. 4$)$.

\section{Discussion}

Autofluorescence of AM is dramatically increased in smokers. It is believed that this results from phagocytosis of particles containing polycyclic aromatic hydrocarbons. This autofluorescence has made it difficult to use FCM for the analysis of specific antigen expression by these cells.

The present study utilizes the technique of crystal violet quenching originally designed by HALLDEN et al. [6] to neutralize the autofluorescence of AM. It has previously been reported that autofluorescence at $525 \mathrm{~nm}$ can be effectively quenched. In the present study, equally effective quenching at $575 \mathrm{~nm}$ was demonstrated. These wavelengths correspond to the fluorescence of FITC and PE, the most common fluorescent dyes used for FCM. Thus, this quenching technique can decrease the background fluorescence, making FCM analysis with these dyes feasible. However, autofluorescence at $675 \mathrm{~nm}$, the emission line for red fluorescence dyes such as Cy5 and allophycocyanin (APC), was increased by the quenching procedure. Thus, this quenching would not be useful when employing red fluorescent dyes. Nevertheless, the quenching procedure should facilitate some two-colour FCM analyses.

In the current report, it was also demonstrated that membrane permeabilization can be used to permit staining of intracellular antigens. CD68 is a widely used macrophage marker in immunohistochemistry. The antibody recognizes a $110 \mathrm{kDa}$ glycoprotein located predominantly on the lysosomal membrane, although a small amount is 

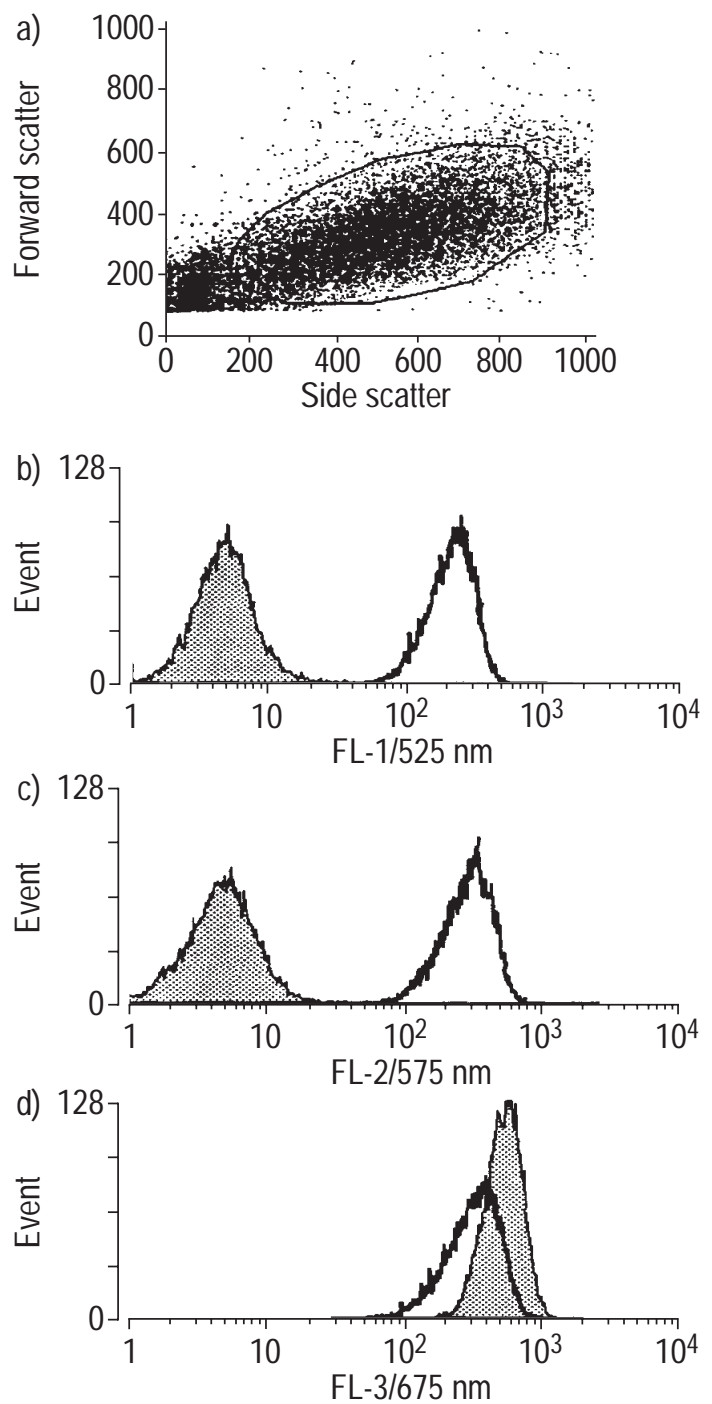
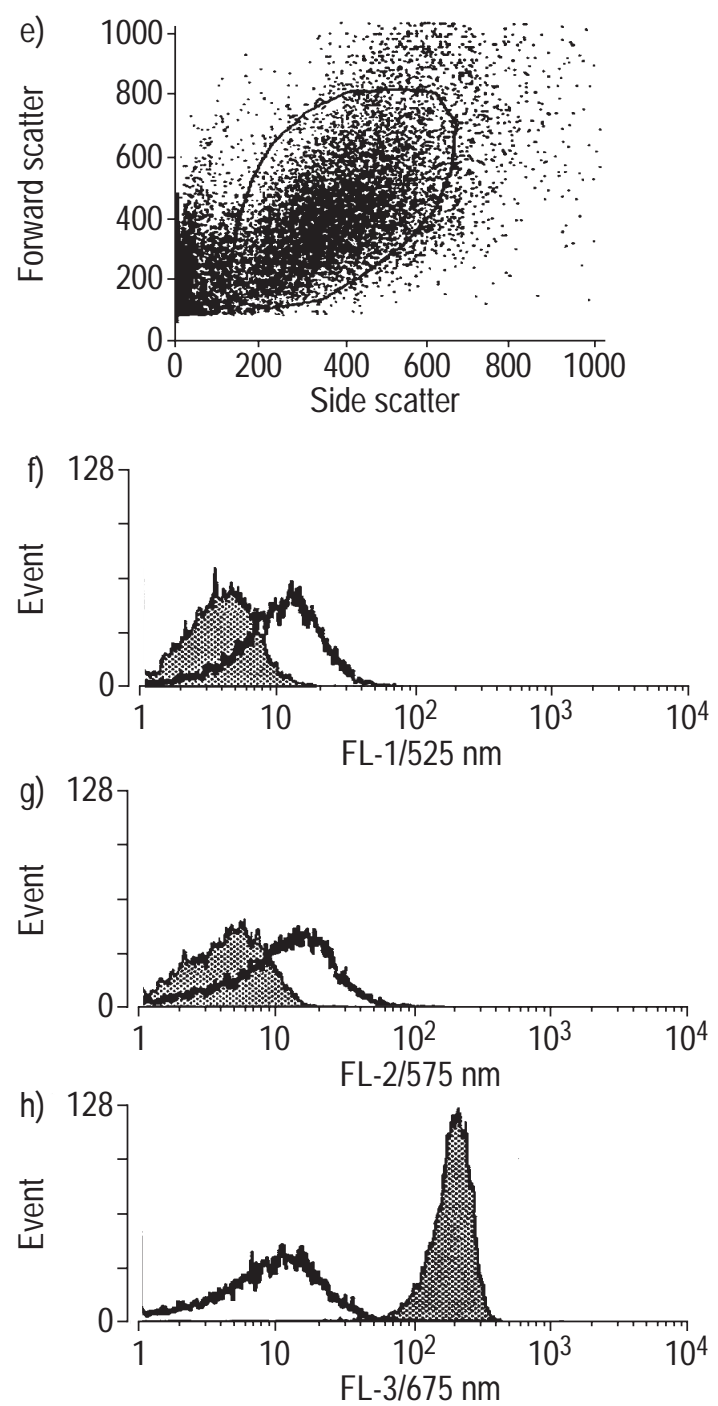

Fig. 2. - Effect of crystal violet quenching. Bronchoalveolar lavage cells were obtained from a 42-yr-old normal smoker with a 30-pack-yr smoking history (a-d) and a 27-yr-old normal nonsmoker (e-h). Alveolar macrophages (AM) were gated as shown in a) and e). AM from a normal smoker had strong autofluorescence (open area) at $525 \mathrm{~nm}$ (fluorescein isothiocyanate/green; b), $575 \mathrm{~nm}$ (phycoerythrin/yellow; c) and $675 \mathrm{~nm}$ (Cy5/red; d) Autofluorescence was minimized by quenching (shaded area) at $525 \mathrm{~nm}$ and $575 \mathrm{~nm}$ (b, c), while it slightly increased (shaded area) at $675 \mathrm{~nm}$ (d). AM from a normal nonsmoker had less autofluorescence (open area; $\mathrm{f}-\mathrm{h}$ ). The level of autofluorescence after quenching (shaded area) for the smoker and nonsmoker at all three bands was almost the same $(\mathrm{b}-\mathrm{d}, \mathrm{f}-\mathrm{h})$.

expressed on the cell surface [4]. Because of the intracellular location of its corresponding antigen, macrophage identification by CD68 was problematic in FCM. However, CD68 can be readily used in FCM with permeabilization $[8,9,11,12]$. The current report demonstrates that following permeabilization, CD68 can be used successfully to study AM by FCM. HLA-DR surface antigen staining was minimally reduced by permeabilization. Although this difference was not significant, the number of subjects evaluated was small. Thus, small alterations owing to the procedure cannot be excluded. It is likely that other epitopes will vary in their stability during the processing method. Thus, appropriate controls will be required to ensure valid quantitative data with other markers.

To demonstrate the effectiveness of this two-colour FCM analysis technique for AM, CD71 expression measured by FCM was compared with data obtained by im- munocytostaining of cytocentrifuge samples. CD71, which corresponds to the transferrin receptor, was chosen because it is located on the cell surface, but is regulated by intracellular iron levels [13]. Approximately $85 \%$ of AM

Table 1. - Effect of crystal violet quenching on autofluorescence of alveolar macrophages by mean fluorescence intensity

\begin{tabular}{lcclcc}
\hline & \multicolumn{2}{c}{ Smokers $(\mathrm{n}=5)$} & & \multicolumn{2}{c}{ Nonsmokers $(\mathrm{n}=5)$} \\
\cline { 2 - 3 } & $\begin{array}{c}\text { Quench } \\
(-)\end{array}$ & $\begin{array}{c}\text { Quench } \\
(+)\end{array}$ & & $\begin{array}{c}\text { Quench } \\
(-)\end{array}$ & $\begin{array}{c}\text { Quench } \\
(+)\end{array}$ \\
\hline $525 \mathrm{~nm}($ FITC) & $17.6 \pm 3.3$ & $0.5 \pm 0.1$ & & $1.4 \pm 0.5$ & $0.3 \pm 0.1$ \\
$575 \mathrm{~nm}($ PE) & $22.5 \pm 5.1$ & $0.3 \pm 0.1$ & & $1.5 \pm 0.3$ & $0.3 \pm 0.1$ \\
\hline
\end{tabular}

Data are shown as mean \pm SD. FITC: fluorescein isothiocyanate; PE: phycoerythrin. 

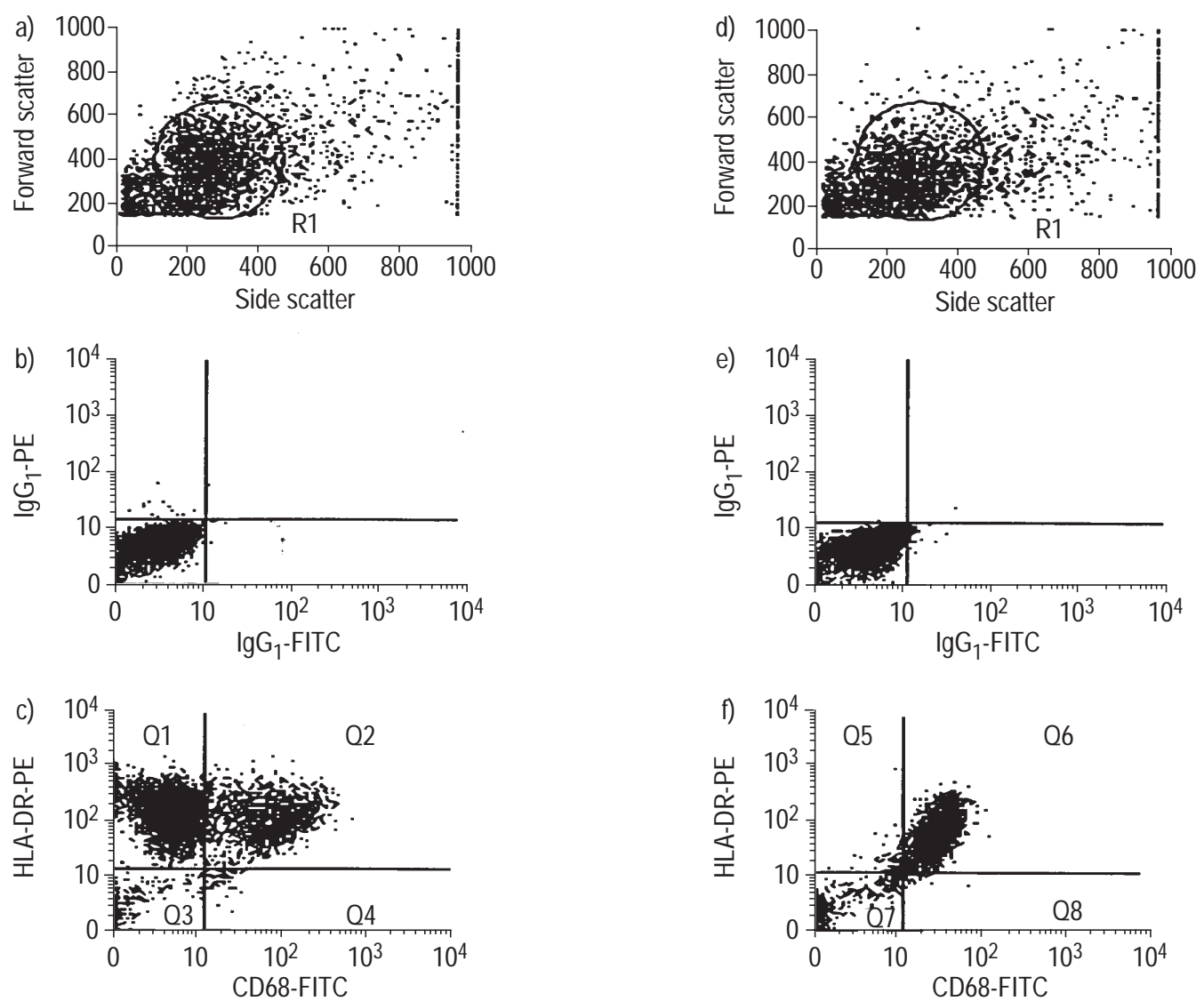

Fig. 3. - Effect of permeabilization on CD68 immunofluorescent staining. Bronchoalveolar lavage cells were from the same source as figure 1 (41-yrold normal current smoker with a 30-pack-yr smoking history). All samples were quenched with crystal violet after immunofluorescent staining to minimize the autofluorescence as described in the Materials and methods. a-c) Cells without permeabilization procedure; d-f) cells with permeabilization procedure. Cells were gated as expected for macrophages by forward and side scatter (a and d). Nonspecific staining by a control antibody (nonspecific mouse immunoglobulin $\mathrm{G}_{1}\left(\mathrm{IgG}_{1}\right)$ ) did not result from the permeabilization procedure (b and e). Only a small percentage of macrophages were positive for CD68 without permeabilization (c) (quadrant (Q)1: 70.6\%; Q2: 25.0\%; Q3: 3.9\%; Q4: 0.5\%). However, most of them were positive for CD68 after the permeabilization procedure (f) (Q5: 3.7\%; Q6: 82.7\%; Q7: 13.2\%; Q8: 0.4\%). Permeabilization did not affect the staining by antibody directed against human leukocyte antigen (HLA)-DR, which is located on the cell surface (c and f). PE: phycoerythrin; FITC: fluorescein isothiocyanate.

from normal nonsmokers express CD71 [7, 14], a result that was confirmed (data not shown). AM from smokers are known to express less CD71 in variable degrees [7], a result also confirmed here (data not shown). Using CD68 to identify the population of macrophages, followed by detection of CD71, it was possible to determine macrophage CD71 expression by FCM. A statistically significant correlation was then observed between the two methods.

The current study is not the first to use FCM for the antigenic analysis of AM. A variety of strategies have been attempted previously to address the problem of autofluorescence. Some investigators have studied only nonsmokers [15-17]. A number of strategies have also been applied to the study of AM obtained from smokers, including the use of long-wave emitting dyes, the use of intracellular quenching and the use of mathematical techniques to attempt to correct for the autofluorescence. VIKSMAN et al. [18] used indirect fluorescence staining with a dye which has peak emission at a higher wavelength $(670 \mathrm{~nm})$ in an attempt to separate the fluorescence emitted by the dye from autofluorescence. Similarly, LOHMEYER et al. [19] reported the use of a long-wave emitting dye (PE/Cy5 tandem; $660 \mathrm{~nm}$ ) together with a mathematical technique to compensate for autofluorescence. WASSERMAN et al. [20] reported a mathematical compensation for the autofluorescence using relative linear median fluorescence intensity (RLMFI) performed on each individual on a patient-bypatient basis. This correction technique was reported to result in a valid collective summation parameter; however, the analysis of individual cells was problematic, thus making cell-by-cell analysis difficult.

Since a larger number of antibodies are available that are conjugated to FITC or PE than to Cy5 or APC, the use of FITC/PE has an advantage for immunostaining. Not only does the method in the current report permit direct immunostaining, but it also enables the study of AM antigen expression on a cell-by-cell basis without special calculations. Thus, the quenching method should have a more general applicability.

The present study demonstrated clear autofluorescence of smokers' AM over the entire spectrum analysed including $675 \mathrm{~nm}$ (red). Other investigators have found sufficiently low autofluorescence at this wavelength to be able to use red emitting dyes. It seems likely that AM autofluorescence at this wavelength will depend on the quantity of smoking. It is noteworthy, therefore, that the smokers in the current study were specifically selected to be very heavy smokers, $>40$ cigarettes $\cdot$ day $^{-1}$. Only $3 \%$ of 


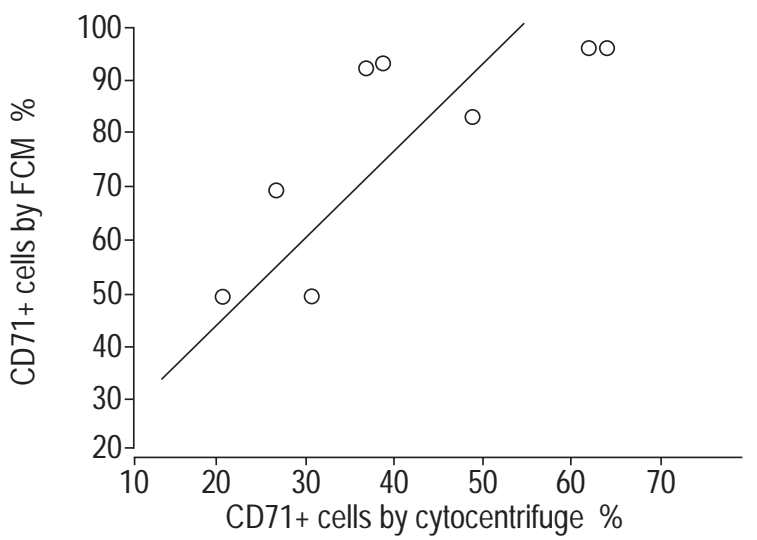

Fig. 4. - Percentage of CD71-positive macrophages measured by flow cytometry (FCM) and immunocytochemistry of cytocentrifuge samples. The results by these two methods showed a statistically significant correlation ( $\mathrm{r}=0.77, \mathrm{p}<0.05$, Pearson's correlation coefficient).

smokers in the USA smoke this amount [21]. It is possible, therefore, that the red autofluorescence observed in the current study would not pose a problem for the use of red emitting dyes, particularly in smokers with a less heavy cigarette consumption.

In summary, crystal violet quenching enabled analysis of the fluorescence staining at $525 / 575 \mathrm{~nm}$. Therefore, it is possible to use antibodies labelled with fluorescein isothiocyanate/phycoerythrin for direct immunofluorescence staining. Moreover, these staining techniques can be performed following a permeabilization procedure and, thus, enable the analysis of intracellular antigens including CD68. This method, therefore, permits analysis by flow cytometry using routine settings for two-colour analysis for fluorescein isothiocyanate and phycoerythrin. Alveolar macrophages obtained from both smokers and nonsmokers can be analysed by this method. In addition, techniques used to analyse alveolar macrophages can also be readily applied to peripheral blood cells studied concurrently. It is likely that this technique will be particularly advantageous for the study of the role of alveolar macrophages in a variety of lung diseases where heavy smokers must, by necessity, be included.

\footnotetext{
Acknowledgements. The authors thank C. Cuszynski and J. Sweeney for their support in cell sorting.
}

\section{References}

1. BAL Cooperative Group Steering Committee. Bronchoalveolar lavage constituents in healthy individuals, idiopathic pulmonary fibrosis, and selected comparison groups. Am Rev Respir Dis 1990; 141: S169-S202.

2. Sköld CM, Hed J, Eklund A. Smoking cessation rapidly reduces cell recovery in bronchoalveolar lavage fluid, while alveolar macrophage fluorescence remains high. Chest 1992; 101: 989-995.

3. Sköld CM, Andersson K, Hed J, Eklund A. Short-term in vivo exposure to cigarette-smoke increases the fluorescence in rat alveolar macrophages. Eur Respir J 1993; 6: 1169-1172.

4. Stockinger H. Cluster report: CD68. In: Knapp W, ed. Leukocyte Typing. IV. New York, Oxford University Press, 1993; pp. 841-843.
5. Striz I, Wang YM, Svarcova I, Trnka L, Sorg C, Costabel $\mathrm{U}$. The phenotype of alveolar macrophages and its correlation with cells in bronchoalveolar lavage. Eur Respir $J$ 1993; 6: 1287-1294.

6. Hallden G, Sköld CM, Eklund A, Forslid J, Hed J. Quenching of intracellular autofluorescence in alveolar macrophages permits analysis of fluorochrome labelled surface antigens by flow cytofluorometry. $J$ Immunol Methods 1991; 142: 207-214.

7. Sköld CM, Lundahl J, Hallden G, Hallgren M, Eklund A. Chronic smoke exposure alters the phenotype pattern and the metabolic response in human alveolar macrophages. Clin Exp Immunol 1996; 106: 108-113.

8. Knapp W, Strobl H, Majdic O. Flow cytometrical analysis of cell-surface and intracellular antigens in leukemia diagnosis. Cytometry 1994; 18: 187-198.

9. Strobl H, Scheinecker C, Csmarits B, Majdic O, Knapp W. Flow cytometrical analysis of intracellular CD68 molecule expression in normal and malignant haemopoiesis. Br J Haematol 1995; 90: 774-782.

10. Rennard SI, Ghafouri M, Thompson AB, et al. Fractional processing of sequential bronchoalveolar lavage to separate bronchial and alveolar sample. Am Rev Respir Dis 1990; 141: 208-217.

11. Hamilton S, Healy M, Corris P, Scott R. An immunohistochemical method for the detection of tumor necrosis factor alpha in cytospins of human bronchoalveolar lavage cells. Histochem J 1995; 27: 487-493.

12. Lan HY, Hutchinson P, Tesch GH, Mu W, Atkins RC. A novel method of microwave treatment for detection of cytoplasmic and nuclear antigens by flow cytometry. $J$ Immunol Meth 1996; 190: 1-10.

13. Schwarting R, Stein H. Cluster report: CD71. In: Knapp W, ed. Leukocyte Typing. IV. New York, Oxford University Press, 1993; pp. 455-460.

14. Hirata T, Bitterman PB, Mornex JF, Crystal RG. Expression of the transferrin receptor gene during the process of mononuclear phagocyte maturation. J Immunol 1986; 136: $1339-1345$.

15. Spurzem JR, Saltini C, Kirby M, Konishi K, Crystal RG. Expression of HLA class II genes in alveolar macrophages of patients with sarcoidosis. Am Rev Respir Dis 1989; 140: 89-94.

16. Buhl R, Jaffe HA, Holroyd KJ, et al. Activation of alveolar macrophages in asymptomatic HIV-infected individuals. J Immunol 1993; 150: 1019-1028.

17. Krombach F, Gerlach JT, Padovan C, et al. Characterization and quantification of alveolar monocyte-like cells in human chronic inflammatory lung disease. Eur Respir J 1996; 9: 984-991.

18. Viksman MY, Liu MC, Schleimer RP, Bochner BS. Application of a flow cytometrical method using autofluorescence and a tandem fluorescent dye to analyze human alveolar macrophage surface markers. J Immunol Meth 1994; 172: 17-24.

19. Lohmeyer J, Friedrich J, Rosseau S, Pralle H, Seeger W. Multiparameter flow cytometrical analysis of inflammatory cells contained in bronchoalveolar lavage fluid. $J$ Immunol Meth 1994; 172: 59-70.

20. Wasserman K, Subklewe M, Pothoff G, Banik N, Schell FE. Expression of surface markers on alveolar macrophages from symptomatic patients with HIV infection as detected by flow cytometry. Chest 1994; 105: 1324-1334.

21. Surgeon General. Changes in smoking behavior and knowledge about determinants. In: Reducing the Health Consequences of Smoking: 25 years of Progress. Rockville, US Dept of Health and Human Services, 1989; pp. 259-378. 